\title{
Some identities for the product of two Bernoulli and Euler polynomials
}

Dae San Kim ${ }^{1}$, Taekyun Kim²*, Sang-Hun Lee ${ }^{3}$ and Young-Hee Kim ${ }^{3}$

* Correspondence: taekyun64@hotmail.com ${ }^{2}$ Department of Mathematics, Kwangwoon University, Seoul 139701, Republic of Korea

Full list of author information is available at the end of the article

\section{Abstract}

Let $\mathbb{P}_{n}$ be the space of polynomials of degree less than or equal to $n$. In this article, using the Bernoulli basis $\left\{B_{0}(x), \ldots, B_{n}(x)\right\}$ for $\mathbb{P}_{n}$ consisting of Bernoulli polynomials, we investigate some new and interesting identities and formulae for the product of two Bernoulli and Euler polynomials like Carlitz did.

\section{Introduction}

The Bernoulli and Euler polynomials are defined by means of

$$
\frac{t}{e^{t}-1} e^{x t}=\sum_{n=0}^{\infty} B_{n}(x) \frac{t^{n}}{n !}, \frac{2}{e^{t}+1} e^{x t}=\sum_{n=0}^{\infty} E_{n}(x) \frac{t^{n}}{n !} .
$$

In the special case, $x=0, B_{n}(0)=B_{n}$ and $E_{n}(0)=E_{n}$ are called the $n$-th Bernoulli and Euler numbers (see [1-17]).

From (1), we note that

$$
B_{n}(x)=\sum_{l=0}^{n}\left(\begin{array}{l}
n \\
l
\end{array}\right) B_{l} x^{n-l}, E_{n}(x)=\sum_{l=0}^{n}\left(\begin{array}{l}
n \\
l
\end{array}\right) E_{l} x^{n-l} .
$$

For $n \geq 0$, we have

$$
\frac{d}{d x} B_{n}(x)=n B_{n-1}(x), \frac{d}{d x} E_{n}(x)=n E_{n-1}(x),
$$

(see $[7,8])$.

By (1), we get the following recurrence for the Bernoulli and the Euler numbers:

$$
B_{0}=1, B_{n}(1)-B_{n}=\delta_{1, n} \text { and } E_{0}=1, E_{n}(1)+E_{n}=2 \delta_{0, n},
$$

where $\delta_{k, n}$ is the Kronecker symbol (see [1-17]).

Thus, from (3) and (4), we have

$$
\int_{0}^{1} B_{n}(x) d x=\frac{\delta_{0, n}}{n+1}, \int_{0}^{1} E_{n}(x) d x=-\frac{2 E_{n+1}}{n+1} .
$$


It is known [12] that

$$
\int_{0}^{A} B_{m_{1}}\left(\frac{x}{a_{1}}\right) \ldots B_{m_{n}}\left(\frac{x}{a_{n}}\right) d x=a_{1}^{1-m_{1}} \ldots a_{n}^{1-m_{n}} \int_{0}^{1} B_{m_{1}}(x) \ldots B_{m_{n}}(x) d x
$$

where $a_{1}, a_{2}, \ldots, a_{n}$ are positive integers that are relatively prime in pairs $A=a_{1} a_{2} \ldots a_{n}$. For $n=2$, there is the formula

$$
\int_{0}^{1} B_{p}(x) B_{q}(x) d x=(-1)^{p+1} \frac{B_{p+q}}{\left(\begin{array}{c}
p+q \\
q
\end{array}\right)}
$$

where $p+q \geq 2$ (see $[3,4]$ ). In $[3,4]$, we can find the following formula for a product of two Bernoulli polynomials:

$$
B_{m}(x) B_{n}(x)=\sum_{r}\left[\left(\begin{array}{c}
m \\
2 r
\end{array}\right) n+\left(\begin{array}{c}
n \\
2 r
\end{array}\right) m\right] \frac{B_{2 r} B_{m+n-2 r}(x)}{m+n-2 r}+(-1)^{m+1} \frac{B_{m+n}}{\left(\begin{array}{c}
m+n \\
n
\end{array}\right)}, \text { for } m+n \geq 2 .
$$

Assume $m, n, p \geq 1$. Then, by (7) and (8), we get

$$
\int_{0}^{1} B_{m}(x) B_{n}(x) B_{p}(x) d x=(-1)^{p+1} p ! \sum_{r}\left[\left(\begin{array}{c}
m \\
2 r
\end{array}\right) n+\left(\begin{array}{c}
n \\
2 r
\end{array}\right) m\right] \frac{(m+n-2 r-1) !}{(m+n+p-2 r) !} B_{2 r} B_{m+n+p-2 r}
$$

(see [4]).

In [8], it is known that for $n \in \mathbb{Z}_{+}$,

$$
B_{n}(x)=\sum_{\substack{k=0 \\
k \neq 1}}^{n}\left(\begin{array}{l}
n \\
k
\end{array}\right) B_{k} E_{n-k}(x)
$$

and

$$
E_{n}(x)=-2 \sum_{l=0}^{n}\left(\begin{array}{l}
n \\
l
\end{array}\right) \frac{E_{l+1}}{l+1} B_{n-l}(x)
$$

Let $\mathbb{P}_{n}=\left\{\sum_{i} a_{i} x^{i} \mid a_{i} \in \mathbb{Q}\right\}$ be the space of polynomials of degree less than or equal to $n$. In this article, using the Bernoulli basis $\left\{B_{0}(x), \ldots, B_{n}(x)\right\}$ for $\mathbb{P}_{n}$ consisting of Bernoulli polynomials, we investigate some new and interesting identities and formulae for the product of two Bernoulli and Euler polynomials like Carlitz did.

\section{Bernoulli identities arising from Bernoulli basis polynomials}

From (1), we note that

$$
\begin{aligned}
e^{x t} & \left.=\frac{1}{t}\left(\frac{t\left(e^{t}-1\right)}{e^{t}-1}\right) e^{x t}\right)=\frac{1}{t} \sum_{n=0}^{\infty}\left(B_{n}(x+1)-B_{n}(x)\right) \frac{t^{n}}{n !} \\
& =\frac{1}{t} \sum_{n=1}^{\infty}\left(B_{n}(x+1)-B_{n}(x)\right) \frac{t^{n}}{n !} \\
& =\sum_{n=0}^{\infty}\left(\frac{B_{n+1}(x+1)-B_{n+1}(x)}{n+1}\right) \frac{t^{n}}{n !} .
\end{aligned}
$$


Thus, from (12), we have

$$
x^{n}=\frac{1}{n+1}\left(B_{n+1}(x+1)-B_{n+1}(x)\right)=\frac{1}{n+1} \sum_{l=0}^{n}\left(\begin{array}{c}
n+1 \\
l
\end{array}\right) B_{l}(x) .
$$

From (13), we note that $\left\{B_{0}(x), B_{1}(x), \ldots, B_{n}(x)\right\}$ spans $\mathbb{P}_{n}$. For $p(x) \in \mathbb{P}_{n}$, let $p(x)=\sum_{k=0}^{n} a_{k} B_{k}(x)$ and $g(x)=p(x+1)-p(x)$. Then we have

$$
g(x)=\sum_{k=0}^{n} a_{k}\left(B_{k}(x+1)-B_{k}(x)\right)=\sum_{k=0}^{n} k a_{k} x^{k-1} .
$$

From (14), we can derive the following Equation (15):

$$
g^{(r)}(x)=\sum_{k=r+1}^{n} k(k-1) \ldots(k-r) a_{k} x^{k-r-1},
$$

where $g^{(r)}(x)=\frac{d^{r} g(x)}{d x^{r}}$ and $r=0,1,2, \ldots, n$. Let us take $x=0$ in (15). Then we have

$$
g^{(r)}(0)=(r+1) ! a_{r+1}
$$

By (16), we get, for $r=1,2, \ldots, n$,

$$
a_{r}=\frac{g^{(r-1)}(0)}{r !}=\frac{1}{r !}\left(p^{(r-1)}(1)-p^{(r-1)}(0)\right) .
$$

Let $0=p(x)=\sum_{k=0}^{n} a_{k} B_{k}(x)$. Then, from (17), we have

$$
a_{r}=\frac{1}{r !} g^{(r-1)}(0)=\frac{1}{r !}\left(p^{(r-1)}(1)-p^{(r-1)}(0)\right)=0 .
$$

From (18), we note that $\left\{B_{0}(x), B_{1}(x), \ldots, B_{n}(x)\right\}$ is a linearly independent set. Therefore, we obtain the following theorem.

Proposition 1 The set of Bernoulli polynomials $\left\{B_{0}(x), B_{1}(x), \ldots, B_{n}(x)\right\}$ is a basis for $\mathbb{P}_{n}$. Let us consider polynomial $p(x) \in \mathbb{P}_{n}$ as a linear combination of Bernoulli basis polynomials with

$$
p(x)=C_{0} B_{0}(x)+C_{1} B_{1}(x)+\cdots+C_{n} B_{n}(x) .
$$

We can write (19) as a dot product of two variables:

$$
p(x)=\left(B_{0}(x), B_{1}(x), \ldots, B_{n}(x)\right)\left(\begin{array}{l}
C_{0} \\
C_{1} \\
\vdots \\
C_{n}
\end{array}\right) .
$$

From (20), we can derive the following equation:

$$
p(x)=\left(1, x, x^{2}, \ldots, x^{n}\right)\left(\begin{array}{ccccc}
1 & b_{12} & b_{13} & \cdots & b_{1 n+1} \\
0 & 1 & b_{23} & \cdots & b_{2 n+1} \\
0 & 0 & 1 & \cdots & b_{3 n+1} \\
\vdots & \vdots & \vdots & \ddots & \vdots \\
0 & 0 & 0 & \cdots & b_{n n+1} \\
0 & 0 & 0 & \cdots & 1
\end{array}\right)\left(\begin{array}{l}
C_{0} \\
C_{1} \\
C_{2} \\
\vdots \\
C_{n}
\end{array}\right),
$$


where $b_{i j}$ are the coefficients of the power basis that are used to determine the respective Bernoulli polynomials. It is easy to show that

$$
B_{0}(x)=1, B_{1}(x)=x-\frac{1}{2}, B_{2}(x)=x^{2}-x+\frac{1}{6}, B_{3}(x)=x^{3}-\frac{3}{2} x^{2}+\frac{1}{2} x, \ldots
$$

In the quadratic case $(n=2)$, the matrix representation is

$$
p(x)=\left(1, x, x^{2}\right)\left(\begin{array}{lll}
1 & -\frac{1}{2} & \frac{1}{6} \\
0 & 1 & -1 \\
0 & 0 & 1
\end{array}\right)\left(\begin{array}{l}
C_{0} \\
C_{1} \\
C_{2}
\end{array}\right) .
$$

In the cubic case $(n=3)$, the matrix representation is

$$
p(x)=\left(1, x, x^{2}, x^{3}\right)\left(\begin{array}{llll}
1 & -\frac{1}{2} & \frac{1}{6} & 0 \\
0 & 1 & -1 & \frac{1}{2} \\
0 & 0 & 1 & -\frac{3}{2} \\
0 & 0 & 0 & 1
\end{array}\right)\left(\begin{array}{l}
C_{0} \\
C_{1} \\
C_{2} \\
C_{3}
\end{array}\right) .
$$

In many applications of Bernoulli polynomials, a matrix formulation for the Bernoulli polynomials seems to be useful.

There are many ways of obtaining polynomial identities in general. Here, in Theorems 2-9, we use the Bernoulli basis in order to express certain polynomials as linear combinations of that basis and hence to get some new and interesting polynomial identities.

Let $I_{m, n}=\int_{0}^{1} B_{m}(x) B_{n}(x) d x$ for $m, n \in \mathbb{Z}_{+}$. Then, by integration by parts, we get

$$
I_{0, n}=I_{m, 0}=0, I_{m, n}=(-1)^{m+n} \frac{B_{m+n}}{\left(\begin{array}{c}
m+n \\
m
\end{array}\right)},(m, n \geq 2) .
$$

For $n \in \mathbb{Z}_{+}$with $n \geq 2$, let us consider the following polynomials in $\mathbb{P}_{n}$ :

$$
p(x)=\sum_{k=0}^{n} B_{k}(x) B_{n-k}(x) \in \mathbb{P}_{n} .
$$

Then, from (25), we have

$$
p^{(r)}(x)=\frac{(n+1) !}{(n-r+1) !} \sum_{k=r}^{n} B_{k-r}(x) B_{n-k}(x)
$$

where $r=0,1,2, \ldots n$.

By Proposition 1, we see that $p(x)$ can be written as

$$
p(x)=\sum_{k=0}^{n} a_{k} B_{k}(x)
$$

From (25) and (27), we note that

$$
a_{0}=\int_{0}^{1} p(t) d t=\sum_{k=0}^{n} I_{k, n-k}=B_{n} \sum_{k=1}^{n-1} \frac{(-1)^{k-1}}{\left(\begin{array}{l}
n \\
k
\end{array}\right)}=B_{n} \frac{\left(1+(-1)^{n}\right)}{n+2}=\frac{2}{n+2} B_{n} .
$$


By (18) and (26), we get

$$
\begin{aligned}
a_{r+1} & =\frac{1}{(r+1) !}\left(p^{(r)}(1)-p^{(r)}(0)\right) \\
& =\frac{(n+1) !}{(r+1) !(n-r+1) !} \sum_{k=r}^{n}\left(B_{k-r}(1) B_{n-k}(1)-B_{k-r} B_{n-k}\right) \\
& =\frac{1}{n+2}\left(\begin{array}{l}
n+r \\
r+1
\end{array}\right) \sum_{k=r}^{n}\left\{\left(\delta_{1, k-r}+B_{k-r}\right)\left(\delta_{1, n-k}+B_{n-k}\right)-B_{k-r} B_{n-k}\right\} \\
& =\frac{1}{n+2}\left(\begin{array}{l}
n+2 \\
r+1
\end{array}\right)\left(B_{n-r-1}+B_{n-r-1}+\delta_{r, n-2}\right) \\
& = \begin{cases}\frac{2}{n+2}\left(\begin{array}{l}
n+2 \\
r+1
\end{array}\right) B_{n-r-1} & \text { if } r \neq n-2 . \\
0 & \text { if } r=n-2 .\end{cases}
\end{aligned}
$$

Therefore, by (25), (27) and (28), we obtain the following theorem.

Theorem 2 For $n \in \mathbb{Z}_{+}$with $n \geq 2$, we have

$$
\sum_{k=0}^{n} B_{k}(x) B_{n-k}(x)=\frac{2}{n+2} \sum_{k=0}^{n-2}\left(\begin{array}{c}
n+2 \\
k
\end{array}\right) B_{n-k} B_{k}(x)+(n+1) B_{n}(x) .
$$

For $n \in \mathbb{Z}_{+}$with $n \geq 2$, let us take polynomial $p(x)$ in $\mathbb{P}_{n}$ as follows:

$$
p(x)=\sum_{k=0}^{n} \frac{1}{k !(n-k) !} B_{k}(x) B_{n-k}(x) \in \mathbb{P}_{n} .
$$

From Proposition 1, we note that $p(x)$ is given by means of Bernoulli basis polynomials:

$$
p(x)=\sum_{k=0}^{n} a_{k} B_{k}(x) \in \mathbb{P}_{n}
$$

By (24), (29) and (30), we get

$$
\begin{aligned}
a_{0} & =\int_{0}^{1} p(t) d t=\sum_{k=0}^{n} \frac{1}{k !(n-k) !} I_{k, n-k}=\frac{2 I_{0, n}}{n !}+\sum_{k=1}^{n-1} \frac{(-1)^{k-1}}{k !(n-k) !\left(\begin{array}{l}
n \\
k
\end{array}\right)} B_{n} \\
& =\frac{B_{n}}{n !} \sum_{k=1}^{n-1}(-1)^{k-1}=\frac{B_{n}}{n !} \frac{\left(1+(-1)^{n}\right)}{2}=\frac{B_{n}}{n !} .
\end{aligned}
$$

From (29), we have that for $r=0,1,2, \ldots, n$,

$$
p^{(r)}(x)=2^{r} \sum_{k=r}^{n} \frac{B_{k-r}(x) B_{n-k}(x)}{(k-r) !(n-k) !} .
$$

By (18), we get

$$
\begin{aligned}
a_{r+1} & =\frac{1}{(r+1) !}\left(p^{(r)}(1)-p^{(r)}(0)\right) \\
& =\frac{2^{r}}{(r+1) !} \sum_{k=r}^{n} \frac{1}{(k-r) !(n-k) !}\left(B_{k-r}(1) B_{n-k}(1)-B_{k-r} B_{n-k}\right) \\
& =\frac{2^{r}}{(r+1) !}\left(\frac{2 B_{n-r-1}}{(n-1-r) !}+\sum_{k=r}^{n} \delta_{1, k-r} \delta_{1, n-k}\right) \\
& =\left\{\begin{array}{cc}
\frac{2^{r+1}}{n !}\left(\begin{array}{c}
n \\
r+1
\end{array}\right) B_{n-r-1} & \text { if } r \neq n-2, \\
0 & \text { if } r=n-2 .
\end{array}\right.
\end{aligned}
$$


Therefore, from (29), (30) and (33), we obtain the following theorem.

Theorem 3 For $n \in \mathbb{Z}_{+}$with $n \geq 2$, we have

$$
\sum_{k=0}^{n}\left(\begin{array}{l}
n \\
k
\end{array}\right) B_{k}(x) B_{n-k}(x)=\sum_{\substack{k=0 \\
k \neq n-1}}^{n} 2^{k}\left(\begin{array}{l}
n \\
k
\end{array}\right) B_{n-k} B_{k}(x) .
$$

Let $n \in \mathbb{Z}_{+}$with $n \geq 2$. Then we consider polynomial $p(x)$ in $\mathbb{P}_{n}$ with

$$
p(x)=\sum_{k=1}^{n-1} \frac{1}{k(n-k)} B_{k}(x) B_{n-k}(x) .
$$

By Proposition 1 , we see that $p(x)$ is written as

$$
p(x)=\sum_{k=0}^{n} a_{k} B_{k}(x)
$$

From (34), we have

$$
\begin{aligned}
a_{0} & =\int_{0}^{1} p(t) d t=\sum_{k=1}^{n-1} \frac{1}{k(n-k)} \int_{0}^{1} B_{k}(t) B_{n-k}(t) d t \\
& =\sum_{k=1}^{n-1} \frac{1}{k(n-k)} \frac{(-1)^{k-1}}{\left(\begin{array}{l}
n \\
k
\end{array}\right)} B_{n}=\left(\frac{1+(-1)^{n}}{n^{2}}\right) B_{n}=\frac{2 B_{n}}{n^{2}} .
\end{aligned}
$$

It is easy to show that for $r=1,2, \ldots, n-1$,

$$
p^{(r)}(x)=2 C_{r} B_{n-r}(x)+(n-1) \cdots(n-r) \sum_{k=r+1}^{n-1} \frac{B_{k-r}(x) B_{n-k}(x)}{(k-r)(n-k)},
$$

where $C_{r}=\frac{1}{n-r} \sum_{j=1}^{r}(n-1) \ldots(n-j+1)(n-j-1) \ldots(n-r)$.

By (17), we get

$$
\begin{aligned}
a_{r+1}= & \frac{1}{(r+1) !}\left(p^{(r)}(1)-p^{(r)}(0)\right) \\
= & \frac{1}{(r+1) !}\left\{2 C_{r}\left(B_{n-r}(1)-B_{n-r}\right)\right. \\
& \left.\quad+(n-1) \ldots(n-r) \sum_{k=r+1}^{n-1} \frac{B_{k-r}(1) B_{n-k}(1)-B_{k-r} B_{n-k}}{(k-r)(n-k)}\right\} \\
= & \frac{2 C_{r}}{(r+1) !} \delta_{r, n-1}+\frac{1}{n}\left(\begin{array}{c}
n \\
r+1
\end{array}\right) \sum_{k=r+1}^{n-1} \frac{B_{k-r} \delta_{1, n-k}+\delta_{1, k-r} B_{n-k}+\delta_{1, k-r} \delta_{1, n-k}}{(k-r)(n-k)} \\
= & \begin{cases}\frac{2}{n(n-r-1)}\left(\begin{array}{c}
n \\
r+1
\end{array}\right) B_{n-r-1} & \text { if } 0 \leq r \leq n-3, \\
\frac{2}{n !} C_{n-1} & \text { if } r=n-2,\end{cases}
\end{aligned}
$$


From the definition of $C_{r}$, we have

$$
\frac{2}{n !} C_{n-1}=\frac{2}{n !} \sum_{i=1}^{n-1} \frac{(n-1) !}{n-i}=\frac{2}{n} \sum_{i=1}^{n-1} \frac{1}{i}=\frac{2}{n} H_{n-1},
$$

where $H_{n}=\sum_{i=1}^{n} \frac{1}{i}$.

Therefore, by (34), (36) and (37), we obtain the following theorem.

Theorem 4 For $n \in \mathbb{Z}_{+}$with $n \geq 2$, we have

$$
\sum_{k=1}^{n-1} \frac{B_{k}(x) B_{n-k}(x)}{k(n-k)}=\frac{2}{n} \sum_{k=0}^{n-2} \frac{1}{n-k}\left(\begin{array}{l}
n \\
k
\end{array}\right) B_{n-k} B_{k}(x)+\frac{2}{n} H_{n-1} B_{n}(x) .
$$

Let $J_{m, n}=\int_{0}^{1} E_{m}(t) E_{n}(t) d t$, for $m, n \in \mathbb{Z}_{+}$. Then we see that

$$
J_{m, n}=\frac{2(-1)^{m-1}}{(n+m+1)\left(\begin{array}{c}
n+m \\
m
\end{array}\right)} E_{n+m+1},(\text { see }[3,4,7,8]) .
$$

Let us take polynomials $p(x)$ in $\mathbb{P}_{n}$ with $p(x)=\sum_{k=0}^{n} E_{k}(x) E_{n-k}(x)$. Then, by Proposition $1, p(x)$ is written as $p(x)=\sum_{k=0}^{n} a_{k} B_{k}(x)$.

It is not difficult to show that

$$
a_{0}=\int_{0}^{1} p(t) d t=\sum_{k=0}^{n} J_{k, n-k}=\frac{2 E_{n+1}}{n+1} \sum_{k=0}^{n} \frac{(-1)^{k-1}}{\left(\begin{array}{l}
n \\
k
\end{array}\right)}=-2 E_{n+1}\left(\frac{1+(-1)^{n}}{n+2}\right)=\frac{-4 E_{n+1}}{n+2}
$$

and

$$
p^{(r)}(x)=\frac{(n+1) !}{(n+1-r) !} \sum_{k=r}^{n} E_{k-r}(x) E_{n-k}(x),(r=0,1,2, \ldots, n) .
$$

By (17) and (39), we get

$$
\begin{aligned}
a_{k} & =\frac{1}{k !}\left(p^{(k-1)}(1)-p^{(k-1)}(0)\right) \\
& =\frac{(n+1) !}{k !(n-k+2) !} \sum_{l=k-1}^{n}\left(E_{l-k+1}(1) E_{n-l}(1)-E_{l-k+1} E_{n-l}\right) \\
& =\frac{\left(\begin{array}{c}
n+2 \\
k
\end{array}\right)}{n+2} \sum_{l=k-1}^{n}\left\{\left(-E_{l-k+1}+2 \delta_{0, l-k+1}\right)\left(-E_{n-l}+2 \delta_{0, n-l}\right)-E_{l-k+1} E_{n-l}\right\} \\
& =-\frac{4\left(\begin{array}{c}
n+2 \\
k
\end{array}\right)}{n+2} E_{n-k+1},
\end{aligned}
$$

where $k=0,1,2, \ldots, n$. Therefore, by (40), we obtain the following theorem.

Theorem 5 For $n \in \mathbb{Z}_{+}$, we have

$$
\sum_{k=0}^{n} E_{k}(x) E_{n-k}(x)=-\frac{4}{n+2} \sum_{k=0}^{n}\left(\begin{array}{c}
n+2 \\
k
\end{array}\right) E_{n-k+1} B_{k}(x) .
$$


Let us take the polynomial $p(x)$ in $\mathbb{P}_{n}$ as follows:

$$
p(x)=\sum_{k=0}^{n} \frac{1}{k !(n-k) !} E_{k}(x) E_{n-k}(x) .
$$

Then, by (41), we get

$$
p^{(r)}(x)=2^{r} \sum_{k=r}^{n} \frac{E_{k-r}(x) E_{n-k}(x)}{(k-r) !(n-k) !},
$$

where $r=0,1,2, \ldots, n$.

By Proposition 1, we see that $p(x)$ can be written as

$$
p(x)=\sum_{k=0}^{n} a_{k} B_{k}(x) .
$$

From (41), (42) and (43), we have

$$
\begin{aligned}
a_{0} & =\int_{0}^{1} p(t) d t=\sum_{k=0}^{n} \frac{1}{k !(n-k) !} J_{k, n-k} \\
& =\frac{2 E_{n+1}}{(n+1) !} \sum_{k=0}^{n}(-1)^{k-1}=-\frac{2 E_{n+1}}{(n+1) !}\left(\frac{1+(-1)^{n}}{2}\right)=\frac{-2 E_{n+1}}{(n+1) !}
\end{aligned}
$$

and

$$
\begin{aligned}
a_{r} & =\frac{1}{r !}\left(p^{(r-1)}(1)-p^{(r-1)}(0)\right) \\
& =\frac{2^{r-1}}{r !} \sum_{k=r-1}^{n} \frac{E_{k-r+1}(1) E_{n-k}(1)-E_{k-r+1} E_{n-k}}{(k-r+1) !(n-k) !} \\
& =\frac{2^{r-1}}{r !}\left(-\frac{2 E_{n-r+1}}{(n-r+1) !}-\frac{2 E_{n-r+1}}{(n-r+1) !}+4 \delta_{n+1, r}\right) \\
& =-\frac{2^{r+1}}{(n+1) !}\left(\begin{array}{c}
n+1 \\
r
\end{array}\right) E_{n-r+1},
\end{aligned}
$$

where $r=1,2, \ldots, n$.

Therefore, by (41), (43) and (45), we obtain the following theorem.

Theorem 6 For $n \in \mathbb{Z}_{+}$, we have

$$
\sum_{k=0}^{n}\left(\begin{array}{l}
n \\
k
\end{array}\right) E_{k}(x) E_{n-k}(x)=-\frac{2}{n+1} \sum_{k=0}^{n} 2^{k}\left(\begin{array}{c}
n+1 \\
k
\end{array}\right) E_{n-k+1} B_{k}(x) .
$$

Let us take

$$
p(x)=\sum_{k=1}^{n-1} \frac{1}{k(n-k)} E_{k}(x) E_{n-k}(x)
$$

in $\mathbb{P}_{n}$. Then, by Proposition $1, p(x)$ is given by means of basis polynomials:

$$
p(x)=\sum_{k=0}^{n} a_{k} B_{k}(x) .
$$


It is easy to show that

$$
\begin{aligned}
a_{0} & =\int_{0}^{1} p(t) d t=\sum_{k=1}^{n-1} \frac{1}{k(n-k)} J_{k, n-k} \\
& =\frac{2 E_{n+1}}{n+1} \sum_{k=1}^{n-1} \frac{1}{k(n-k)} \frac{(-1)^{k-1}}{\left(\begin{array}{l}
n \\
k
\end{array}\right)}=\frac{2\left(1+(-1)^{n}\right)}{n^{2}(n+1)} E_{n+1}=\frac{4 E_{n+1}}{n^{2}(n+1)}
\end{aligned}
$$

and

$$
p^{(k)}(x)=2 C_{k} E_{n-k}(x)+(n-1) \ldots(n-k) \sum_{l=k+1}^{n-1} \frac{E_{l-k}(x) E_{n-l}(x)}{(l-k)(n-l)},(k=1,2, \ldots, n-1)
$$

where $C_{k}=\frac{1}{(n-k)} \sum_{j=1}^{k}(n-1) \ldots(n-j+1)(n-j-1) \ldots(n-k)$.

By the same method, we get

$$
\begin{aligned}
a_{k}= & \frac{1}{k !}\left(p^{(k-1)}(1)-p^{(k-1)}(0)\right) \\
=\frac{1}{k !}\left\{2 C_{k-1}\left(E_{n-k+1}(1)-E_{n-k+1}\right)\right. & \\
& \left.\quad+(n-1) \ldots(n-k+1) \sum_{l=k}^{n-1} \frac{E_{l-k+1}(1) E_{n-l}(1)-E_{l-k+1} E_{n-l}}{(l-k+1)(n-l)}\right\} \\
= & -\frac{4 C_{k-1}}{k !} E_{n-k+1} .
\end{aligned}
$$

From the construction of $C_{k}$, we note that

$$
\begin{aligned}
\frac{C_{k-1}}{k !} & =\frac{1}{k !(n-k+1)} \sum_{j=1}^{k-1}(n-1) \ldots(n-j+1)(n-j-1) \ldots(n-k+1) \\
& =\frac{1}{k !(n-k+1)} \sum_{j=1}^{k-1} \frac{(n-1) !}{(n-k) !(n-j)}=\frac{\left(\begin{array}{l}
n \\
k
\end{array}\right)}{n(n-k+1)} \sum_{j=1}^{k-1} \frac{1}{n-j} \\
& =\frac{\left(\begin{array}{l}
n \\
k
\end{array}\right)}{n(n-k+1)}\left(\sum_{j=1}^{n-1} \frac{1}{j}-\sum_{j=1}^{n-k} \frac{1}{j}\right)=\frac{\left(\begin{array}{l}
n \\
k
\end{array}\right)}{n(n-k+1)}\left(H_{n-1}-H_{n-k}\right) .
\end{aligned}
$$

Therefore, by the same method, we obtain the following theorem.

Theorem 7 For $n \in \mathbb{Z}_{+}$with $n \geq 2$, we have

$$
\sum_{k=1}^{n-1} \frac{E_{k}(x) E_{n-k}(x)}{k(n-k)}=\frac{4 E_{n+1}}{n^{2}(n+1)}-\frac{4}{n} \sum_{k=1}^{n} \frac{\left(\begin{array}{l}
n \\
k
\end{array}\right)}{n-k+1}\left(H_{n-1}-H_{n-k}\right) E_{n-k+1} B_{k}(x) .
$$

Let

$$
T_{m, n}=\int_{0}^{1} B_{m}(t) E_{n}(t) d t, \quad \text { for } m, n \in \mathbb{Z}_{+}
$$


From (47), we have that

$$
T_{m, 0}=\int_{0}^{1} B_{m}(t) d t=\frac{\delta_{0, m}}{m+1} \quad \text { and } \quad T_{0, n}=\int_{0}^{1} E_{n}(t) d t=-\frac{2 E_{n+1}}{n+1} .
$$

For $m, n \in \mathbb{N}$, we have

$$
T_{m, n}=\frac{2(-1)^{m}}{(m+n+1)\left(\begin{array}{c}
m+n \\
m
\end{array}\right)} \sum_{l=m+1}^{m+n}(-1)^{l}\left(\begin{array}{c}
m+n+1 \\
l
\end{array}\right) B_{l} E_{n+m+1-l} .
$$

Let us consider the following polynomial in $\mathbb{P}_{n}$ :

$$
p(x)=\sum_{k=0}^{n} B_{k}(x) E_{n-k}(x) .
$$

For $n \in \mathbb{N}$ with $n \geq 2$, by Proposition $1, p(x)$ is given by

$$
p(x)=\sum_{k=0}^{n} a_{k} B_{k}(x) .
$$

From (49) and (50), we note that

$$
\begin{aligned}
a_{0} & =\int_{0}^{1} p(t) d t=T_{0, n}+\sum_{k=1}^{n-1} T_{k, n-k}+T_{n, 0} \\
& =-\frac{2 E_{n+1}}{n+1}+\frac{2}{n+1} \sum_{k=1}^{n-1} \sum_{l=k+1}^{n}(-1)^{k+l} \frac{\left(\begin{array}{c}
n+1 \\
l
\end{array}\right)}{\left(\begin{array}{c}
n \\
k
\end{array}\right)} B_{l} E_{n+1-l} .
\end{aligned}
$$

For $k=0,1,2, \ldots, n$, we have

$$
\begin{aligned}
p^{(k)}(x) & =(n+1) n \ldots(n+2-k) \sum_{l=k}^{n} B_{l-k}(x) E_{n-l}(x) \\
& =\frac{(n+1) !}{(n-k+1) !} \sum_{l=k}^{n} B_{l-k}(x) E_{n-l}(x) .
\end{aligned}
$$

By (17), we get

$$
\begin{aligned}
a_{k} & =\frac{1}{k !}\left(p^{(k-1)}(1)-p^{(k-1)}(0)\right) \\
& =\frac{(n+1) !}{k !(n-k+2) !} \sum_{l=k-1}^{n}\left(B_{l-k+1}(1) E_{n-l}(1)-B_{l-k+1} E_{n-l}\right) \\
& =\frac{\left(\begin{array}{c}
n+2 \\
k
\end{array}\right)}{n+2} \sum_{l=k-1}^{n}\left\{\left(B_{l-k+1}+\delta_{1, l-k+1}\right)\left(-E_{n-l}+2 \delta_{0, n-l}\right)-B_{l-k+1} E_{n-l}\right\} \\
& =\frac{\left(\begin{array}{c}
n+2 \\
k
\end{array}\right)}{n+2}\left(-2 \sum_{l=k-1}^{n} B_{l-k+1} E_{n-l}-E_{n-k}+2 B_{n-k+1}+2 \delta_{n, k}\right) .
\end{aligned}
$$


Therefore, by (49), (50) and (53), we obtain the following theorem.

Theorem 8 For $n \in \mathbb{Z}_{+}$with $n \geq 2$, we have

$$
\begin{aligned}
& \sum_{k=0}^{n} B_{k}(x) E_{n-k}(x) \\
& =-\frac{2 E_{n+1}}{n+1}+\frac{2}{n+1} \sum_{k=1}^{n-1} \sum_{l=k+1}^{n}(-1)^{k+l} \frac{\left(\begin{array}{c}
n+1 \\
l
\end{array}\right)}{\left(\begin{array}{l}
n \\
k
\end{array}\right)} B_{l} E_{n+1-l}+(n+1) B_{n}(x) \\
& +\frac{1}{n+2} \sum_{k=1}^{n-2}\left(\begin{array}{c}
n+2 \\
k
\end{array}\right)\left(-2 \sum_{l=k-1}^{n} B_{l-k+1} E_{n-l}-E_{n-k}+2 B_{n-k+1}\right) B_{k}(x) .
\end{aligned}
$$

For $n \in \mathbb{N}$ with $n \geq 2$, let us take $p(x)=\sum_{k=0}^{n} \frac{B_{k}(x) E_{n-k}(x)}{k !(n-k) !}$ in $\mathbb{P}_{n}$. Then we have

$$
p^{(k)}(x)=2^{k} \sum_{l=k}^{n} \frac{1}{(l-k) !(n-l) !} B_{l-k}(x) E_{n-l}(x) .
$$

From Proposition 1, we note that $p(x)$ can be written as

$$
p(x)=\sum_{k=0}^{n} a_{k} B_{k}(x) .
$$

Thus, by (55), we get

$$
\begin{aligned}
a_{0} & =\int_{0}^{1} p(t) d t=\sum_{k=0}^{n} \frac{1}{k !(n-k) !} T_{k, n-k} \\
& =\frac{T_{0, n}}{n !}+\sum_{k=1}^{n-1} \frac{T_{k, n-k}}{k !(n-k) !}+\frac{T_{n, 0}}{n !} \\
& =-\frac{2 E_{n+1}}{(n+1) !}+\frac{2}{(n+1) !} \sum_{k=1}^{n-1} \sum_{l=k+1}^{n}(-1)^{k+l}\left(\begin{array}{c}
n+1 \\
l
\end{array}\right) B_{l} E_{n+1-l} .
\end{aligned}
$$

From (17), we note that

$$
\begin{aligned}
a_{k} & =\frac{1}{k !}\left(p^{(k-1)}(1)-p^{(k-1)}(0)\right) \\
& =\frac{2^{k-1}}{k !} \sum_{l=k-1}^{n} \frac{B_{l-k+1}(1) E_{n-l}(1)-B_{l-k+1} E_{n-l}}{(l-k+1) !(n-l) !} \\
& =\frac{2^{k-1}}{k !}\left(\sum_{l=k-1}^{n} \frac{-2 B_{l-k+1} E_{n-l}}{(l-k+1) !(n-l) !}-\frac{E_{n-k}}{(n-k) !}+\frac{2 B_{n-k+1}}{(n-k+1) !}+2 \delta_{n, k}\right) .
\end{aligned}
$$

Therefore, by (54), (55) and (57), we obtain the following theorem. 
Theorem 9 For $n \in \mathbb{N}$ with $n \geq 2$, we have

$$
\begin{aligned}
& \sum_{k=0}^{n}\left(\begin{array}{l}
n \\
k
\end{array}\right) B_{k}(x) E_{n-k}(x) \\
& =-\frac{2 E_{n+1}}{n+1}+\frac{2}{n+1} \sum_{k=1}^{n-1} \sum_{l=k+1}^{n}(-1)^{k+l}\left(\begin{array}{c}
n+1 \\
l
\end{array}\right) B_{l} E_{n+1-l} \\
& +\sum_{k=1}^{n-2}\left(\begin{array}{c}
-\frac{2^{k}\left(\begin{array}{c}
n+1 \\
k
\end{array}\right)}{n+1} \sum_{l=k-1}^{n}\left(\begin{array}{c}
n-k+1 \\
n-l
\end{array}\right) B_{l-k+1} E_{n-l}-2^{k-1}\left(\begin{array}{l}
n \\
k
\end{array}\right) E_{n-k} \\
+\frac{2^{k}\left(\begin{array}{c}
n+1 \\
k
\end{array}\right)}{n+1} B_{n-k+1}
\end{array}\right) B_{k}(x)+2^{n} B_{n}(x) .
\end{aligned}
$$

For $n \in \mathbb{N}$ with $n \geq 2$, let us consider the polynomial $p(x)=\sum_{k=1}^{n-1} \frac{B_{k}(x) E_{n-k}(x)}{k(n-k)}$ in $\mathbb{P}_{n}$.

From Proposition 1 , we note that $p(x)$ can be written as $p(x)=\sum_{k=0}^{n} a_{k} B_{k}(x)$. Then the $k$-th derivative of $p(x)$ is given by

$$
p^{(k)}(x)=C_{k}\left(B_{n-k}(x)+E_{n-k}(x)\right)+(n-1) \ldots(n-k) \sum_{l=k+1}^{n} \frac{B_{l-k}(x) E_{n-l}(x)}{(l-k)(n-l)}
$$

where $k=1,2, \ldots, n-1$ and

$$
C_{k}=\frac{1}{n-k} \sum_{j=1}^{k}(n-1)(n-2) \ldots(n-j+1)(n-j-1) \ldots(n-k) .
$$

In addition,

$$
p^{(n)}(x)=\left(p^{(n-1)}(x)\right)^{\prime}=\left(C_{n-1}\left(B_{1}(x)+E_{1}(x)\right)\right)^{\prime}=2 C_{n-1}=2(n-1) ! H_{n-1} .
$$

From (17), we note that

$$
\begin{aligned}
& a_{k}=\frac{1}{k !}\left(p^{(k-1)}(1)-p^{(k-1)}(0)\right) \\
& =\frac{C_{k-1}}{k !}\left\{\left(B_{n-k+1}(1)-B_{n-k+1}\right)+\left(E_{n-k+1}(1)-E_{n-k+1}\right)\right\} \\
& +\frac{(n-1) \ldots(n-k+1)}{k !} \sum_{l=k}^{n-1} \frac{1}{(l-k+1)(n-l)}\left(B_{l-k+1}(1) E_{n-l}(1)-B_{l-k+1} E_{n-l}\right) \\
& =\frac{C_{k-1}}{k !}\left(-2 E_{n-k+1}+\delta_{1, n-k+1}\right)+\frac{\left(\begin{array}{l}
n \\
k
\end{array}\right)}{n}\left(\sum_{l=k}^{n-1} \frac{-2 B_{l-k+1} E_{n-l}}{(l-k+1)(n-l)}-\frac{E_{n-k}}{n-k}\right) .
\end{aligned}
$$

It is easy to show that

$$
\begin{aligned}
a_{0} & =\int_{0}^{1} p(t) d t=\sum_{k=1}^{n-1} \frac{1}{k(n-k)} T_{k, n-k} \\
& =\frac{2}{(n+1) n(n-1)} \sum_{k=0}^{n-2} \frac{(-1)^{k+1}}{\left(\begin{array}{c}
n-2 \\
k
\end{array}\right)} \sum_{l=k+2}^{n}(-1)^{l}\left(\begin{array}{c}
n+1 \\
l
\end{array}\right) B_{l} E_{n+1-l} .
\end{aligned}
$$


Therefore, from (59) and (60), we have

$$
\begin{aligned}
& \sum_{k=1}^{n-1} \frac{1}{k(n-k)} B_{k}(x) E_{n-k}(x) \\
& =\frac{2}{n\left(n^{2}-1\right)} \sum_{k=0}^{n-2} \sum_{l=k+2}^{n}(-1)^{k+l+1} \frac{\left(\begin{array}{c}
n+1 \\
l
\end{array}\right)}{\left(\begin{array}{c}
n-2 \\
k
\end{array}\right)} B_{l} E_{n+1-l} \\
& \quad+\sum_{k=1}^{n-2}\left\{\frac{-2}{n(n-k+1)}\left(\begin{array}{l}
n \\
k
\end{array}\right)\left(H_{n-1}-H_{n-k}\right) E_{n-k+1}\right. \\
& \left.\quad+\frac{1}{n}\left(\begin{array}{l}
n \\
k
\end{array}\right)\left(-2 \sum_{l=k}^{n-1} \frac{B_{l-k+1} E_{n-l}}{(l-k+1)(n-l)}-\frac{E_{n-k}}{n-k}\right)\right\} B_{k}(x)+\frac{2}{n} H_{n-1} B_{n}(x) .
\end{aligned}
$$

\section{Acknowledgements}

The authors would like to express their deep gratitudes to the referees for their valuable suggestions and comments.

\section{Author details}

${ }^{1}$ Department of Mathematics, Sogang University, Seoul 121-742, Republic of Korea ${ }^{2}$ Department of Mathematics, Kwangwoon University, Seoul 139-701, Republic of Korea ${ }^{3}$ Division of General Education, Kwangwoon University, Seoul 139-701, Republic of Korea

\section{Authors' contributions}

All authors contributed equally to the manuscript and typed, read and approved the final manuscript.

\section{Competing interests}

The authors declare that they have no competing interests.

Received: 10 April 2012 Accepted: 2 July 2012 Published: 2 July 2012

\section{References}

1. Araci, S, Erdal, D, Seo, Jj: A study on the fermionic $p$-adics $q$-integral representation on $\mathbb{\Xi}_{p}$ associated with weighted $q$ Bernstein and $q$-Genocchi polynomials. Abstr Appl Anal 2011, 10 (2011). (Article ID 649248)

2. Bayad, A: Modular properties of elliptic Bernoulli and Euler functions. Adv Stud Contemp Math. 20(3):389-401 (2010)

3. Carlitz, L: Product of two Eulerian polynomials. Math Mag. 36, 37-41 (1963). doi:10.2307/2688134

4. Carlitz, L: Note on the integral of the product of several Bernoulli polynomials. J Lond Math Soc. 34, 361-363 (1959). doi:10.1112/jlms/s1-34.3.361

5. Cangul, IN, Kurt, V, Ozden, H, Simsek, Y: On the higher-order w-q-Genocchi numbers. Adv Stud Contemp Math. 19, 39-57 (2009)

6. Cenkci, M, Simsek, Y, Kurt, V: Multiple two-variable $p$-adic $q$-L-function and its be-havior at $s=0$. Russ J Math Phys. 15 , 447-459 (2008). doi:10.1134/S106192080804002X

7. Kim, DS, Kim, T: A study on the integral of the product of several Bernoulli polynomials. Rocky Mountain Journal of Mathematics. (in press)

8. Kim, DS, Dolgy, DV, Kim, HM, Lee, SH, Kim, T: Integral formula of Bernoulli polynomials. Adv Stud Contemp Math. 22(2):190-199 (2012)

9. Kim, T: An identity of symmetry for the generalized Euler polynomials. J Comput Anal Appl. 13(7):1292-1296 (2011)

10. Kim, T: Some identities for the Bernoulli, the Euler and the Genocchi numbers and polynomials. Adv Stud Contemp Math. 20, 23-28 (2010)

11. Kim, T: $q$-Bernoulli numbers and polynomials associated with Gaussian binomial coefficients. Russ J Math Phys. 15, $51-57(2008)$

12. Mordell, L: Integral formulas of arithmetic characters.J Lond Math Soc. 33, 371-375 (1957)

13. Ozden, $H$, Simsek, Y: A new extension of $q$-Euler numbers and polynomials related to their interpolation functions. Appl Math Lett. 21(9):934-939 (2008). doi:10.1016/..aml.2007.10.005

14. Ryoo, CS: Some relations between twisted $q$-Euler numbers and Bernstein polynomials. Adv Stud Contemp Math. 21(2):217-223 (2011)

15. Ryoo, CS: Some identities of the twisted $q$-Euler numbers and polynomials associated with $q$-Bernstein polynomials. Proc Jangjeon Math Soc. 14(2):239-248 (2011)

16. Simsek, Y: Complete sum of products of $(h, q)$-extension of Euler polynomials and numbers. J Diff Equ Appl. 16(11):1331-1348 (2010). doi:10.1080/10236190902813967 
17. Simsek, Y: Theorems on twisted L-function and twisted Bernoulli numbers. Adv Stud Contemp Math. 11(2):205-218 (2005)

doi:10.1186/1687-1847-2012-95

Cite this article as: Kim et al: Some identities for the product of two Bernoulli and Euler polynomials. Advances in Difference Equations 2012 2012:95.

Submit your manuscript to a SpringerOpen ${ }^{\odot}$ journal and benefit from:

- Convenient online submission

- Rigorous peer review

- Immediate publication on acceptance

- Open access: articles freely available online

- High visibility within the field

- Retaining the copyright to your article

Submit your next manuscript at $\gg$ springeropen.com 